\title{
Optimal self-avoiding paths in dilute random medium
}

\author{
F.Seno ${ }^{1}$, A.L. Stella ${ }^{2}$ and C. Vanderzande ${ }^{3}$ \\ (1) INFM-Dipartimento di Fisica, Università di Padova, Italy \\ (2) INFM-Dipartimento di Fisica e Sezione INFN, Università di Padova, Italy \\ (3) Department Wiskunde Natuurkunde Informatica, Limburgs Universitair Centrum, 3590 \\ Diepenbeek, Belgium
}

(March 31, 2017)

By a new type of finite size scaling analysis on the square lattice, and by renormalization group calculations on hierarchical lattices we investigate the effects of dilution on optimal undirected self-avoiding paths in a random environment. The behaviour of the optimal paths remains the same as for directed paths in undiluted medium, as long as forbidden bonds are not exceeding the percolation threshold. Thus, overhanging configurations do not alter the standard self-affine directed polymer scaling regime, even above the directed threshold, when they become unavoidable.

When dilution reaches the undirected threshold, the optimal path becomes fractal, with fractal dimension equal to $D_{\text {min }}$, the dimension of the minimal length path on percolation cluster backbone.

In this regime the optimal path energy fluctuation, $\overline{\Delta E}$, can be ascribed entirely to minimal length fluctuations, and satisfies $\overline{\Delta E} \propto L^{\omega}$, with $\omega=1.02 \pm 0.06$ in $2 d, L$ being the Euclidean distance. Hierarchical lattice calculations confirm that $\omega$ is also the exponent of the leading scaling correction to $\bar{E} \propto L^{D_{\min }}$. Upon approaching threshold, the probability, $\mathcal{R}$, that the optimal path does not stick entirely on the minimal length one, obeys $\mathcal{R} \sim(\Delta p)^{\rho}$, with $\rho \sim 1.0 \pm 0.05$ on hierarchical lattices. Such behaviour could be characteristic of the crossover to fractal regime. Transfer matrix results on square lattice show that a similar full sticking does not occur for directed 
paths at the directed percolation threshold. 


\section{INTRODUCTION}

The problem of directed paths in a random potential has attracted a lot of interest because of its connection with issues like domain walls in random ferromagnets [1], interface growth [2], fracture and failure phenomena [3] and magnetic flux lines in high-temperature ceramic superconductors 仹.

At zero temperature the interface separating oppositely magnetized phases in a $2 d$ random Ising ferromagnet is given by a minimal energy path on the dual lattice. Upon averaging over randomness the path has self-affine geometry with roughness exponent $\zeta=2 / 3[\mathbb{1}]$. The energy fluctuates about average with a longitudinal size dependence described by the exponent $\omega=1 / 3$ [1,2]. Up to now, models exhibiting this kind of behaviour have always been studied with the simplifying restriction of allowing only directed path configurations ( directed polymer in random medium: DPRM). This means that the path, besides being self-avoiding, can not develop overhangs. Such a restriction is not expected to be essential for obtaining a path with self-affine geometry at large length scales. On the other hand, removal of the directedness constraint on the path must be crucial, e.g., for those situations in which disorder becomes of such a nature to induce crossover from self-affine to fractal geometry.

In this paper we address the problem of determining the scaling properties of optimal (undirected) self-avoiding paths, under the effect of a percolative, geometrical disorder which forbids a given fraction of lattice edges to the path. Such disorder, associated to the existence of percolative voids, is considered in combination with the usual energy randomness of the remaining accessible bonds. Models of this type, but directed, were considered recently by Balents and Kardar [6] and are expected to have applications to fractures of porous materials [7] and interface growth of poisoned Eden models [8,9].

In the context of domain walls in random ferromagnets we can imagine a $2 d$ Ising problem in which a fraction, $p$, of nearest neighbour spins has an extremely strong, essentially infinite, ferromagnetic exchange, while the others have a distribution of random couplings with finite 
limits and variance.

At $T=0$ the domain wall separating oppositely magnetized phases will of course not pass through the duals of infinite exchange bonds, which as a result are excluded from the wall.

One should notice that, for a configuration of exchange strengths in which walls fully excluding infinitely strong couplings are not allowed, the minimal energy of the domain wall would be infinite as well. Our choice is to exclude such case from the statistics. So, the optimization problem looses meaning for us in case $p$ exceeds the percolation threshold. An operative way to realize the exclusion could consist in using appropriate boundary conditions, able to push the interface to stick on one of the edges of the sample as soon as the otherwise optimal path should cross infinitely strong bonds.

Another case in which the optimal interface has to develop within a restricted space is when the system undergoing a phase transition is hosted in the pores of inert structures like aerogels. A possibility of describing these situations is by means of spin models in a suitably diluted and random environment [12].

For the above interfaces, the optimal configuration is thus that in which energy is minimized by passing only through duals of the weakest among accessible random bonds, which amount to a fraction $1-p$ of the total.

In ref [6] it was discovered that a directed path in such an environment remains of the standard type, with $\zeta=2 / 3$ and $\omega=1 / 3$, as long as $(1-p)>p_{\text {cd }}$, where $p_{\text {cd }}$ is the directed percolation threshold for allowed bonds. Right at $(1-p)=p_{\text {cd }}$ the optimal path has to pass through the incipient infinite cluster $(I I C)$ of directed percolation and its properties change drastically. Indeed one finds [6] $\zeta=0.61 \pm 0.04$ and $\omega \sim 0.50 \pm 0.01$, which suggest $\zeta=\nu_{\perp} / \nu_{\|}, \nu_{\perp}$ and $\nu_{\|}$being the correlation length exponents of directed percolation in $2 d$ [10].

A main issue related to such a transition can be formulated as follows.

Suppose we release the constraint of directedness for the paths. We know that, in this case, the directed percolation threshold marks only the point below which one can not find 
anymore directed paths within clusters of allowed bonds. A lower threshold, $p_{c}<p_{\mathrm{cd}}$, marks now the complete disappearance of connecting paths with overhangs. It is interesting to investigate the nature of the optimal path for $p_{c}<(1-p)<p_{\text {cd }}$. Indeed, this is a situation in which only overhanging configurations are allowed by the disorder, and one can not exclude new scalings to arise. Indeed the possible effect of overhangs exclusion on asymptotic behaviour is a very debated and controversial issue, especially in the context of interface growth models, which are closely related to DPRM [5].

A further interesting problem is that of determining the precise behaviour of the optimal path when it has to pass through the backbone of the (undirected) $I I C$, which is a fractal structure. It is of particular interest to determine the new scalings characterizing geometry and energetics of the path also in this condition. Besides establishing how the laws of the self-affine regime generalize to the fractal one, such a study should also elucidate the possible connection between path scaling properties and backbone geometry.

As we will discuss below, clear cut answers to the above and other, related questions are often very difficult to give if one works only with paths on $2 d$ regular lattice. The reason is that, as soon as the walks are not directed, the standard statistical treatment, based on the transfer matrix, becomes extremely time and memory consuming and does not allow easy extrapolation of finite size results.

This is the main reason why, in the recent past, most of the issues we are facing here were essentially not addressed.

In this paper we produce a main effort towards implementation of a finite size scaling (FSS) analysis based on transfer matrix of self-avoiding paths in geometrically and energetically random $2 d$ environments.

At the same time, in view of the above mentioned difficulties, we try to gain additional insight by an extensive investigation of realizations of our models on suitable hierarchical lattices. On such lattices one has the great advantage that renormalization group (RG) recursion equations can be written exactly. By numerical methods, such equations can be iterated long enough to allow satisfactory control of asymptotic scaling properties. 
The results we present for hierarchical lattices should be considered as important and substantial complements, and sometimes substitutes, of findings in the Euclidean lattice.

The present paper is organized as follows. In the next section, we introduce our model on Euclidean lattice and its hierarchical realizations. Renormalization group results for hierarchical lattice are also presented and discussed there. The third section is devoted to the transfer matrix approach on square lattice. Results concern both undirected and directed self-avoiding walks (SAW). A summary of the results, together with concluding remarks are given in the last, fourth section.

\section{OPTIMAL SELF-AVOIDING PATHS ON HIERARCHICAL LATTICES.}

As anticipated in the introduction, we want to consider a path optimization problem which can be formulated as follows in the case of a $2 d$, square lattice. With an independently and identically distributed probability, $p$, each lattice edge can be assigned the role of inaccessible (e.g., infinite energy) bond in a given random configuration. To the remaining, accessible bonds, $b$, finite positive energies $E_{b}$ are assigned according to an independent distribution $\mathcal{P}\left(E_{b}\right)$. For each random configuration obtained in this way we then try to determine the minimal energy self-avoiding paths (if any) connecting (through accessible bonds) two given points at distance $L$. The energy of a self-avoiding path $W$ is of course given by

$$
E_{W}=\sum_{b \in W} E_{b}
$$

Of particular interest is to determine the optimal path energy fluctuations with respect to the average over all random configurations. Such fluctuations can be described by

$$
\overline{\Delta E}=\left(\overline{\left(E_{\min }-\overline{E_{\min }}\right)^{2}}\right)^{1 / 2}
$$

where, in a given configuration,

$$
E_{\text {min }}=\min _{W}\left(\sum_{b \in W} E_{b}\right)
$$


and the bar indicates average over all configurations.

In the case of directed paths in random, undiluted medium, it is found that $\overline{\Delta E} \sim L^{\omega}$ for $L \rightarrow \infty$ [1]. This scaling is related to the geometrical behaviour of the path in a simple way. Indeed, for directed paths, this geometry is always self-affine, with transverse fluctuations with respect to the straight path of length $L$, growing as $L_{\perp} \sim L^{\zeta}$, $\zeta$ being a roughness exponent. By linking energy to length fluctuations [1] one can also derive

$$
\omega=2 \zeta-1
$$

The $\omega$ exponent determines also the leading scaling correction to the average optimal path energy, according to

$$
\bar{E} \sim L\left(1+c L^{\omega-1}\right) \text { for } L \rightarrow \infty
$$

In ref. [6] the same problem with dilution defined above, but restricted to directed paths, was considered at finite temperature. In that case each walk $W$ is weighed by a Boltzmann factor $\exp \left(-E_{W} / T\right)$, and all admissible walks enter in the statistical averages for any given configuration. Rather convincing evidence was obtained in ref [6] that, as long as (1 p) $>p_{\text {cd }}$, the directed paths behave with exponents $\omega=2 / 3$ and $\zeta=2 / 3$ satisfying eq. (2.4). When $(1-p)=p_{\text {cd }}$, the behaviour is still self-affine, but with different exponents $\omega=0.50 \pm 0.01$ and $\zeta=0.61 \pm 0.04 \sim \nu_{\perp} / \nu_{\|}, \nu_{\perp}$ and $\nu_{\|}$being the correlation length exponents of directed percolation [10]. In this case eq. (2.4) is not satisfied anymore, and the result for $\zeta$ can be understood in the sense that, for $(1-p)=p_{\mathrm{cd}}$, the path is limited by the backbone of the directed percolation $I I C$. This limitation of an otherwise standard walk $(\omega=1 / 3, \zeta=2 / 3)$ has been indicated as possibly responsible of the violation of eq. (2.4) [6]. Our goal in this work is to understand how all this possibly modifies when considering undirected optimal paths, and to describe the new physics setting in when dilution is pushed further than directed percolation threshold.

In the next section we illustrate several results obtained by applying transfer matrix methods to our undirected model and to a variant of that treated in ref. [6]. 
However, the difficulty of the subject suggests to approach the main physical issues also in the context of hierarchical models. Indeed, for these models the RG approach can give quite accurate results at a very reasonable computational cost. If not a good approximation, such results should at least provide a rather complete, qualitative picture of the behaviour of models on Euclidean lattice. This is of outmost importance in our case.

Let us consider a hierarchical lattice obtained by following the iteration scheme illustrated in Fig. 1a. The resulting lattice is a diamond with a diagonal bond (DDHL). So far, diamonds without diagonal (DHL) (Fig. 1b) have been always considered for the description of directed paths in random media [14]. However, on a DHL all paths joining the extrema have the same length $\left(2^{n}\right)$ at the $n$-th stage of construction, and there is no way of introducing a distinction between "directed" and "undirected" paths on them.

Here a self-avoiding path joining the DDHL ends will be considered directed if, by following its development through the various stages of the construction, it never passes through the diagonal of any diamond. In other words, directed paths on DDHL are those which could be hosted by a DHL. As a matter of fact the DDHL was already used recently to represent the properties of SAW in random environment [16]. While this reference focuses on the properties in the regime corresponding to the standard criticality of SAW on regular lattice, here we are concentrating on what can be called the stretched regime, and on the effects of directedness removal on it.

On a DDHL the directed percolation threshold is at $(1-p)=p_{\mathrm{cd}}=(\sqrt{5}-1) / 2$. The undirected threshold is at $(1-p)=p_{c}=1 / 2$. For the renormalization group treatment we consider the joint energy and length probability distribution for optimal paths crossing the DDHL at stage $n$. If $N(W)=\sum_{b \in W} 1$ is the number of steps of $W$, we indicate this probability by $P_{n}(E, N)$. With our initial condition clearly:

$$
P_{0}(E, N)=\delta_{N, 1}[(1-p) P(E)+p \delta(E-\infty)]
$$

where $\mathcal{P}$ is the energy probability distribution of accessible bonds. With our assumption that no bond with infinite energy can be crossed by the optimal path, averages based on eq. 
(2.6) must of course ignore the second term in brackets, and include division by $(1-p)$ in order to restore normalization.

$P_{n+1}$ can be constructed from $P_{n}$ as follows:

$$
P_{n+1}(E, N)=\sum_{\left\{N_{\alpha}\right\}} \int \Pi_{\alpha=1}^{5}\left[d E_{\alpha} P_{n}\left(E_{\alpha}, N_{\alpha}\right) \delta\left(E-\min _{W} \sum_{\beta \in W} E_{\beta}\right) \delta_{N, \sum_{\gamma \in W_{\min }}} N_{\gamma}\right]
$$

where $\alpha$ numbers the five bonds of a diamond cell with diagonal (Fig.1a), $W$ are the 4 distinct self-avoiding paths crossing the cell, and $W_{\min }$ indicates among them that for which $\sum_{\beta \in W} E_{\beta}$ is minimal.

Of course, also $P_{n}$ will contain a part proportional to $\delta(E-\infty)$. By construction, the coefficient of this term, which has to be excluded in the discussion of the allowed optimal paths, is proportional to $p^{(n)}$, the $n$-iterated of the RG transformation

$$
p^{\prime}=\left(2 p^{3}-5 p^{2}+2 p+2\right) p^{2}
$$

for bond percolation on the DDHL.

Thus, for $p<p_{c}$, the recursion (2.7) drives $P_{n}$ towards $p=0$, i.e. to a situation where excluded, $E=\infty$, bonds disappear. For $p>p_{c}$, only excluded bonds survive for $n \rightarrow \infty$, and no optimal path exists, in the sense explained above. For $p=p_{c}=1 / 2$, the concentration of forbidden bonds remains fixed under iteration and only their energy distribution evolves.

Iteration of eq. (2.7) can not be performed exactly. A simple and accurate numerical strategy consists in iterating a large sample of paths distributed in energy and length according to $P_{n}$. In the interest of easier computational tractability, we choose

$$
\mathcal{P}(E)=q \delta(E-1)+(1-q) \delta(E-2)
$$

Our normal choice will be $q=1 / 2$, in case we want to treat a finite width distribution. $q=0$ will apply to the case of zero width. As far as $q=1 / 2$ is concerned, we expect, and explicitly verified, that different choices of $q$ or of the energy values are not relevant. We expect the $q=1 / 2$ asymptotic results to belong to the same universality class as those obtained with more standard distributions on continuous interval. Our $\mathcal{P}$ offers the computational advantage that optimal path energies remain positive integers. 
The initial sample of paths is a large collection of $\left(E_{\mathrm{i}}^{(0)}, N_{\mathrm{i}}^{(0)}\right), i=1,2, \ldots i_{\max }\left(i_{\max } \leq\right.$ $10^{6}$ ), randomly generated according to (2.6). Groups of 5 pairs are extracted from the sample, and each couple is associated to one of the 5 bonds of the DDHL cell. For each group the optimal path across the cell is identified, and its total energy, and length are stored in the new ensemble as $\left(E_{\mathrm{i}}^{(1)}, N_{\mathrm{i}}^{(1)}\right)$, and are distributed according to $P_{1}$. This can be iterated several times before serious statistical problems due to limited sampling arise.

The knowledge of $P_{n}$ allows to determine quantities like $\overline{E_{\min }}, \overline{\Delta E}$ or the average path length, $\bar{N}$, as a function of the initial conditions and of $L=2^{n}$, the (longitudinal) length of the lattice. We could normally handle $n \leq 20$ in our calculations. Of course the procedure just described can be applied also to the same model on DHL, or on more complicated lattices. The percolation threshold for DHL, $(\sqrt{5}-1) / 2=0.6180 \ldots$, coincides with $p_{\mathrm{cd}}$ for DDHL.

For our model on DDHL we first considered the case $(1-p)>p_{\text {cd }}$, with energy disorder specified by $q=1 / 2$. The asymptotic regime here is not modified, with respect to the DHL case, by the presence of undirected configurations. Indeed we find $\omega=\log (1.230 \ldots) / \log (2) \sim 0.30$, which coincides, within the numerical accuracy, with the value first obtained in ref [13]. On DHL this $\omega$ nicely corresponds to the DPRM value $\omega=1 / 3$ on Euclidean lattice.

Assuming validity of (2.4) one gets also a roughness exponent $\zeta \sim 0.65$, obviously close to the DPRM value $2 / 3$. This result, for $(1-p)>p_{\mathrm{cd}}$ is certainly consistent with what is expected to happen on Euclidean lattices, but was never explicitly verified so far, to our knowledge. At $(1-p)=p_{\mathrm{cd}}$ the path on DDHL behaves differently from that on DHL. Indeed, for DHL, $(1-p)=p_{\text {cd }}$ is the percolation threshold, below which the optimization problem looses meaning. One can verify that, at $(1-p)=p_{\text {cd }}, \omega \sim 0.49 \pm 0.02$ on DHL, whereas $\omega \sim 0.30$ remains for DDHL.

$\omega \sim 0.50$ was already found for $T>0$ on DHL in ref. [6], where it was shown to be in agreement with the transfer matrix result for directed paths on square lattice, $\omega=0.50 \pm 0.01$. 
Our finding for DDHL shows that the directed threshold is not felt at all by the undirected optimal path. Moreover, we find that, in the whole region $p_{c}<(1-p) \leq p_{\text {cd }}$, $\omega$ keeps the value 0.30 . This means that, even if optimal path configurations must now contain overhangs, these are not able to affect the large scale behaviour. A further confirmation of the fact that the optimal path on DDHL has to be considered self-affine for $p_{c}<(1-p) \leq 1$, comes from a computation of the fractal dimension, $D$, of the path, based on $\bar{N} \sim\left(2^{n}\right)^{D}$. With very high accuracy we find $D=1$ on the whole range. Notice that on DHL, which in our context represents the implementation of the directedness constraint, $D=1$ holds by construction.

We conjecture therefore that a self affine DPRM behaviour with $\omega=1 / 3$ and $\zeta=2 / 3$ should hold on Euclidean lattice as long as $(1-p)>p_{c}$.

Of particular interest is the case $(1-p)=p_{c}=1 / 2$ on DDHL. For this threshold case we find $\omega=1.04 \pm 0.05$. Assuming validity of eq. (2.4), $\omega=1$ would correspond to $\zeta=1$, the limiting value where self-affinity changes into fractality.

The fractal character of the path is demonstrated by $D=1.085 \pm 0.005$. In fact the optimal path at threshold is constrained to develop within the backbone of the infinite incipient percolation cluster, and we can try to identify $D$ among the fractal dimensions of the backbone on DDHL. A natural candidate is the dimension $D_{\min }$ of the minimal length path across the backbone. One way to test this is that of comparing $D$ values for disordered bond energies $(q=1 / 2)$, and for a case with no disorder ( $q=0$, for example). In the latter case, the optimal path has to coincide with the minimal length path, thus $D=D_{\text {min }}$ must hold. Remarkably, at $1-p=p_{c}$ we find no appreciable differences in the $D$ and $\omega$ values for $q=1 / 2$, and $q=0$. The fact that also $\omega$ remains unchanged by putting $q=0$, means that energy fluctuations in this regime are dominated by the geometrical backbone disorder: thus $\omega$ has the geometrical meaning of describing the fluctuations of minimal chemical distance within the backbone. The presence or absence of energy disorder in the backbone bonds does not affect the value of $\omega$, which is just a direct manifestation of the IIC geometry.

A further confirmation that $D=D_{\text {min }}$ comes from a direct exact $\mathrm{RG}$ evaluation of $D_{\text {min }}$ 
on DDHL, which gives $D_{\min }=\log (17 / 8) / \log 2=1.089$. This determination can be done straightforwardly by appropriately weighing, at $p=p_{c}$, the lengths of all possible path crossing a percolating cell and performing the average.

At first sight $\omega=1$ seems to qualify as a natural exponent for optimal paths having a fractal geometry in view of the relation (2.4). Notice, however, that the validity of eq. (2.4) is not a priori obvious: at the directed percolation threshold directed paths on Euclidean lattice do not satisfy such relation, for example.

Also as an indication of the predictive value of this kind of model calculations, it is worth checking the robustness of $\omega$ with respect to modifications of the hierarchical lattice. We used other lattices, with more complicated cells, reproducing features of the square lattice and allowing distinction between directed and undirected thresholds. Two examples are reported in Fig. 1c and Fig. 1d.

With similar, somewhat more heavy RG calculations, we could confirm the scenario described above for the DDHL. In particular, at the percolation thresholds we could determine $\omega=1.02 \pm 0.05$ and $\omega=1.01 \pm 0.05$, rather consistent with the DDHL result. We should expect the results for $\omega$ in the case of the lattices in fig. 1c and $1 \mathrm{~d}$ to represent better approximation of the Euclidean exponent.

To better elucidate the meaning of $\omega$ in the case of fractal optimal path, we also checked how eq. (2.5) changes in this case. Our data for $\overline{E_{\min }}$ could be fitted very well by the form:

$$
\overline{E_{\min }}(L) \sim a L^{D_{\min }}+b L^{\omega} \quad L \gg 1
$$

with $D_{\min }=1.08$ and, $\omega=1.01 \pm 0.03$ and $L=2^{n}$. Eq. (2.10), is consistent with an asymptotic distribution

$$
Q_{n}(E)=\sum_{N} P_{n}(E, N)=\frac{1}{L^{\omega}} f\left(\frac{E-E_{\min }(L)}{L^{\omega}}\right) \quad(E<\infty)
$$

of optimal paths energie widths. Thus $\omega$ should be the only scaling dimension for energy.

A stressed above, the fact that, at $(1-p)=p_{c}, D$ and $\omega$ remain the same for $q=0$, indicates that $D=D_{\min }$, and that energy fluctuations must be fully ascribed to optimal path 
length fluctuations. In other words, at large scales, the optimal path must stick completely on the minimal length path.

It is interesting to contrast this behaviour with the situation at $(1-p)>p_{c}$. To this purpose one can introduce a quantity $\mathcal{R}_{n}$, which represents the probability that, on a DDHL of length $2^{n}$, the optimal path deviates from the minimal length one. This quantity can be easily computed by checking, at each RG iteration, the fraction of optimal paths for which the choice of minimizing $E$ is not leading to a simultaneous minimization of $N$. $\mathcal{R}_{n}$ can be easily followed in different regimes. We find that, as long as $(1-p)>p_{c}, \mathcal{R}_{n}$ approaches a nonzero limiting value for $n \rightarrow \infty$. This indicates that in the self-affine scaling regime deviations from the minimal path always occur. On the other hand,the limiting value of $\mathcal{R}_{n}$ appears to approach 0 for $(1-p) \searrow p_{c}$, consistent with what we found above. A fit of the behaviour of $\mathcal{R} \equiv \lim _{n \rightarrow \infty} \mathcal{R}_{n}$ as a function of $(1-p)$ gives:

$$
\mathcal{R} \simeq\left[1-p-p_{c}\right]^{\rho} \quad \text { for }(1-p) \searrow p_{c}
$$

with $\rho=1.0 \pm 0.05$. This is a new critical exponent associated to the crossover from self-affine to fractal regimes.

Similar values of $\rho$ could be estimated for the lattices in Fig. 1c and 1d. The quantity $\mathcal{R}$ with its behaviour (2.12) offers a further characterization of the transition from self-affine to fractal regime. This transition can be interpretated as a full adsorption undergone by the polymer on the minimal length path. It would be quite interesting to obtain an estimate of $\rho$ for paths on Euclidean lattice. Unfortunately such an estimate did not turn out to be feasible with the methods we describe in the following section.

Another interesting issue is whether a quantity like $\mathcal{R}$ behaves in similar way for directed walks at the directed percolation threshold. Unfortunately, since on DHL all paths have the same length, a computation of $\mathcal{R}$ on DHL for directed paths does not make sense . However, in the next section we compute this quantity for directed paths on square lattice ( direction along one of the lattice axes and solve the issue). 


\section{FINITE SIZE SCALING APPROACH TO OPTIMAL SELF-AVOIDING PATHS ON 2D SQUARE LATTICE}

We now turn to a study of self-avoiding paths on an Euclidean square lattice. We call $x$ the abscissa and $y$ the ordinate. The paths have one end fixed in $(0,0)$. As in the previous section, each bond can be accessible or not, and to the accessible bonds random energies are associated, consistently with (2.6). In our numerical calculations we will again use (2.9) with $q=1 / 2$.

For a given $x=t$ we consider all possible paths joining the origin with the $x=t$ vertical axis (we "cut" the paths at the first intersection with this axis, i.e. the intersection at shortest " chemical" distance from the origin).

For each $y$ on the $x=t$ axis we consider the minimal energy of the paths arriving at $y$, $E_{\min }(t, y)$. The minimal energy fluctuations can be estimated by [11]:

$$
\Delta E(t)=E_{\min }(t, 0)-E_{\min }(t)
$$

where

$$
E_{\min }(t)=\min _{y}\left\{E_{\min }(t, y)\right\}
$$

and $y_{\min }(t)$ indicates the $y$ at which the minimum energy is reached. In the diluted case $((1-p)<1)$ the deviation $(3.1)$ could be meaningless in case no path arrives at $(t, 0)$. To avoid this one can choose, e.g., the point closest to the origin instead of $(t, 0)$ in each configuration. Alternatively, as in the previous section, one can take for $\Delta E(t)$ the root mean square variance over all $E_{\min }(t, y)$. The general ideas on path optimization, and the results of the previous section let us expect the following scalings:

$$
\begin{gathered}
\overline{\Delta E} \sim t^{\omega} \quad t \rightarrow \infty \\
\overline{\left|y_{\min }(t)\right|} \sim t^{\zeta} \quad t \rightarrow \infty
\end{gathered}
$$

where again the bars indicate averages with respect to random bond configurations. 
So far, we assumed that the self-avoiding paths can occupy the whole half-plane $0 \leq$ $x,-\infty<y<+\infty$.

Of course it is hopeless to deal with all such paths. In order to determine the exponents in (3.3) and (3.4) we propose here a finite size scaling (FSS) strategy which exploits the possibility of considering the problem restricted to rectangular boxes, $0 \leq x \leq t, 1 \leq y \leq L$, with $L$ finite (and relatively small), and $t$ ranging up to values much larger than $L$. Optimal self-avoiding path within such boxes should obey finite size version of (3.3) and (3.4). For example

$$
\overline{\Delta E(t, L)} \sim t^{\omega} F\left(\frac{t^{\zeta}}{L}\right) \quad t \rightarrow \infty \quad L \rightarrow \infty
$$

$F$, consistently with eq.(3.2), should satisfy

$$
\lim _{z \rightarrow 0} F(z)=\text { const }
$$

and, in view of the fact that, at fixed $L$ and for $t^{\zeta} \gg L, \overline{\Delta E}$ should approach a $t$ independent saturation value,

$$
F(z) \sim z^{-\omega / \zeta}
$$

This property follows from the fact that $\overline{\Delta E}$ is defined as average over bond configurations of a quantity which is a difference between $\underline{t w o}$ energies in each configuration. If, on the contrary, we would define $\overline{\Delta E}$ as the root mean square variance of $E_{\min }(t)$ over all configurations we would get $\overline{\Delta E}(t, L) \sim t^{\frac{1}{2}}$. This last result is a consequence of the central limit theorem. Indeed, for $t^{\zeta} \gg L$, the box becomes essentially one-dimensional and $E_{\min }(t)$ can be seen as the sum of many independent random terms, corresponding to different segments along the $L$ axis.

Eq. (3.7) can be used to estimate $\omega$ and $\zeta$ by extrapolating results for relatively small $L$. If we call $N(t)$ the number of steps of the minimal energy path within the rectangle $0 \leq x \leq t, 1 \leq y \leq L$ one expects the following FSS scaling form for the averaged $N(t)$

$$
\overline{N(t)} \sim t^{D} G\left(\frac{t^{\zeta}}{L}\right)
$$


where $G(z)$ approaches a constant for $z$ approaching zero, so that $\overline{N(t)} \sim t^{D}$ for $L \gg t^{\zeta}$.

On the other hand, for $t^{\zeta} \gg L$, we must have $\overline{N(t)} \sim t$ 円, so that $G(z) \sim z^{\frac{1-D}{\zeta}}$.

Thus, for $t^{\zeta} \gg L \gg 1$

$$
\overline{N(t)} \sim t L^{\frac{D-1}{\zeta}}
$$

In case the path is fractal, we expect $D>1$ and $\zeta=1$, while for the self-affine case $D=1$ and $\zeta<1$, in eq. (3.9). As a consequence $\overline{N(t)} \sim t L^{D-1}$ in the fractal regime and $\overline{N(t)} \sim t$ in the self-affine regime. The behaviour of $\bar{N}$ for $t^{\zeta} \gg L$ thus allows a direct estimate of $D$.

For each bond configuration in a box the optimal self-avoiding path energy and its length have been computed exactly by a transfer matrix technique. We used the well known transfer matrix approach for self-avoiding walks introduced by Derrida [21]. Due to the randomness, symmetry properties of the transfer matrix disappear and in this way the size of the matrix is larger then in the non-random case. Average over all bond configurations could be performed on a Monte Carlo basis. At $1-p=p_{c}$. the number of paths reaching the $x=t$-axis decreases as a power in $t$, so that self-averaging cannot be used. Therefore a great number of random configurations has to be used to obtain accurate statistics (we used up to $4.10^{6}$ configurations). This limited our calculations to the ranges $L \leq 9$ and $t \leq 100$.

For the small values of $L$ considered here no direct estimate of $\omega$ or $D$ in the regime $t^{\zeta} \ll L$ was possible. We were however able to get accurate estimates of $\omega / \zeta$ and $D$ from the saturation value of $\overline{\Delta E}$ and the large $t$ behaviour of $\overline{N(t)}$, respectively. Fig 2 shows a typical result for $\overline{\Delta E(t)}$ for $L=9$ at $1-p=p_{c}$ with $q=1 / 2$.

Our results very clearly indicate $D=1$ in the whole region where $p_{c}<1-p<1$. For large values of $1-p, \omega / \zeta$ also clearly goes to its DPRM value of $1 / 2$ for large $L$. Unfortunately in the region $p_{c}<1-p<p_{c d}$ we are unable to draw definite conclusions on the value of $\omega / \zeta$. The numerical estimates are much larger then the DPRM-value, but decrease slowly with

1 This follows from the fact that $t \leq N(t) \leq L t$ and thus must be linear in $t$ for large $t$. 
increasing $L$. It is unclear whether the estimates will converge to $1 / 2$ or to a higher value. The results on the hierarchical lattice lead us to believe there is no change in $\omega / \zeta$ at $p_{c d}$ and that the higher values found here are due to crossover-effects. To solve completely the issue on square lattice would require a computational effort beyond our present possibilities.

Of particular interest are our determinations of $D$, and $\omega / \zeta$ right at $(1-p)=p_{c}=1 / 2$. Our results for $\mathrm{D}$ now clearly indicate that the optimal path becomes a fractal with a dimension $D=1.16 \pm 0.04$ with much confidence. In view of the discussion and results in the previous section, we should consider this as a determination of $D_{\min }$ for the backbone of the $I I C$ in $2 d$. This dimension is not know exactly. Only a few numerical results exist,which are not particularly consistent [18]. Our determination is not far from the independent ones found in the literature for $D_{\min }$, and we think it should not be less accurate.

Finally, we extrapolated with reasonable accuracy $\omega / \zeta=1.02 \pm 0.06$. Because we are now in a fractal regime we get $\zeta=1$ and thus $\omega=1.02 \pm 0.06$, surprisingly consistent with the values for hierarchical models considered in the previous section.

As mentioned in the previous section, we did not succeed in getting meaningful extrapolations for $\mathcal{R}$ with our FSS approach to self avoiding paths. Obviously the efficiency of the transfer matrix approach is much higher with directed paths. Much attention has been devoted in the literature to the case in which the directedness is oriented along the diagonal of a square lattice [1,22]. In such condition it is possible to construct a recursion relation which has a very simple structure. In fact, due to the directedness, at each site only two bonds are merging and we can reduce the optimization procedure to just a choice between two possible energies. For a given realization of randomness, the energy of the optimal polymer configuration connecting the origin to all possible end points is easily computed by using iteratively the recursion relation.

Although the number of possible paths grows as $2^{t}$, the relatively simple structure of the optimization procedure makes this approach extremely efficient and powerful. However, to the purpose of this research paper, it is crucial to have a distinction between the paths which minimize the energy and the ones which have the minimal geometrical length. The square 
lattice with directedness along the diagonal does not allow this distinction because all the paths have the same length. We then must put the directedness along one of the principal axis, e.g. $\hat{x}$, in order to avoid this drawback. As for the undirected case the paths have to be hosted in a strip of width $L$ along the direction perpendicular to the directed one.

The recursion relation we must iterate becomes:

$$
E_{\min }(y, x+1)=\min _{1 \leq k \leq L}\left\{E_{\min }(k, x)+\sum_{i=k, y} E_{b \perp}(i, x)+E_{b \|}(y, x)\right\}
$$

where $E_{b \perp}$ and $E_{b \|}$ represent the random energies which are attributed to the bonds perpendicular or parallel to the oriented axis, respectively.

Eq. (3.10) is more time and memory consuming than the one used for the diagonal case, but its directed nature allows us to find the optimal solution still in a polynomial time.

We have already pointed out for the undirected case that the restriction of working with the size constraint measured by $L$ can be partially overtaken from the possibility of estimating the scaling exponents from the saturation values of $\Delta E$ by using equation (3.5). However for this case we can still reach very high values of the strip width $L$ (of the order of thousands), so we can also extract directly the critical indices $\zeta$ and $\omega$ through a standard log-log plot of the suitable quantities. In all our calculations we tried to use both methods in order to minimize statistical errors. Nevertheless these errors are a little bit bigger than those usually found for the proper directed problem in view of the shorter times we are obliged to use.

Our first step was to investigate the best energy paths above the percolation threshold of this model: $p_{c}=0.555 \pm 0.002$ [15].

As expected we found a quite good agreement with the DPRM exponents $\omega=1 / 3$ and $\zeta=2 / 3$. By working in boxes up to $L=3000$ and $t=3000$ we found:

$$
\zeta=0.65 \pm 0.04 \quad \omega=0.34 \pm 0.02
$$

Then we investigated the properties of the best energy paths at the directed percolation threshold. This becomes quite computationally intensive because a large fraction of the 
configurations must be thrown away before a percolating cluster is found. We could obtain good statistics for samples up to $L=1000$ and $t=1000$ and we obtained:

$$
\omega=0.51 \pm 0.03 \quad \zeta=0.64 \pm 0.06
$$

The results are very consistent with those obtained in ref. [6]. If we can not discriminate whether $\zeta$ is really different from the DPRM value $2 / 3$, we can certainly state that $\omega>1 / 3$ as expected from the calculation on the DHL.

In analogy with the work we did on the DDHL lattice we then tried to study the difference between the best energetic path and the shortest one. For each random configuration and for any time step we computed the position of both paths and we stored the difference $\Delta y$ between the two. We have also defined and analyzed a quantity $\mathcal{R}(t)$ which represents the probability that after $t$ steps the optimal path deviates from the shortest one.

In this case the results do not agree with those obtained for undirected paths on DDHL. We have clearly found that the quantity $\mathcal{R}(t)$ does not go to zero at $p_{c}$. This conclusion is also confirmed by plots of the ratio between the number of bonds in common between the two kind of optimal paths and the total number of bonds as a function of $t$. Moreover we

have seen that the average transversal displacement $(\overline{\Delta y(t)})$ grows with $t$ following the law:

$$
\overline{\Delta y(t)} \sim t^{\bar{\zeta}}=t^{0.67 \pm 0.07}
$$

This result and the fact that $\zeta$ can not be bigger than $\bar{\zeta}$ support the conclusion that $\bar{\zeta}=\zeta$

The above results show that a scaling exponent like $\rho$ can not be defined at a threshold where the path remains self-affine.

\section{CONCLUSIONS}

In this work we carried on a systematic analysis of self-avoiding walks in random environment with a fraction of forbidden bonds. We wanted to establish up to which extent the 
removal of the directedness constraint and the replacement of directed by isotropic percolation can lead to new physics for the optimal path solution.

Our analysis was partly based on hierarchical lattice RG calculations. At the same time, in section 3 we set up a new efficient FSS scheme for the evaluation of optimal path properties on $2 \mathrm{~d}$ Euclidean lattice. Beyond the specific results obtained here, this type of scheme could have other applications in the field. E.g., its generalization to finite temperature is rather straightforward. One could also use it for determining properties of SAW in random environment referring to regimes different from the one treated in the present paper. For example, if the length of the walk is properly controlled by a step fugacity, one expects that the SAW can be made critical in the standard sense of polymer statistics [16]. Working on DDHL, we also verified that simultaneous use of positive and negative energy values in the distribution (2.9), upon varying $q$, allows to identify a $T=0$ fixed point at the border between the domains of attraction of our minimal length fixed point and a similar one, dominated by maximal length paths on the backbone. This border multicritical point turns out to have the scaling properties expected for self-avoiding walks on percolation clusters on DDHL [16].

Thus our FSS methods could be used in order to study problems like the SAW on percolation clusters 16, 17,23. An interesting methodological indication we got here is that a transfer matrix based FSS analysis of such problems can give sensible results, especially as far as fractal dimensions are concerned. Of course the full description of the crossover between stretched minimal length and maximal length regimes would be extremely time consuming in view of the necessity of testing many $q$ and $L$ values.

Our calculation here show that the standard DPRM behaviour [1,2] of directed paths applies also to the undirected case as long as $(1-p)>p_{c}$. Thus, the presence of overhanging configurations does not alter the large scale behaviour of the path, even for $p_{c}<(1-p)<p_{\mathrm{cd}}$, when backward turns must be present in all path configurations. This is indication of particular robustness of the standard DPRM behaviour. A priori one could not exclude that the directed percolation threshold could mark a transition to a different self-affine scaling 
regime. Our results seem to rule out such possibility.

A further characterization of the $(1-p)>p_{c}$ regime is given through the quantity $\mathcal{R}$, which measures the probability of detouchment of the optimal path from the minimal length walk. In the self-affine regime $\mathcal{R}$ is always non-zero; thus a non-zero fraction of optimal paths deviates from the minimal length one. For $(1-p)$ approaching $p_{c}, \mathcal{R}$ approaches zero, with a $\rho$ exponent close to unity on "two dimensional" hierarchical lattices. The fact that $\mathcal{R}$ approaches zero at threshold could be peculiar of cases in which the path geometry crosses over from self-affine to fractal. For directed paths at the directed threshold $p_{c d}, \mathcal{R}$ does not become zero, indicating that, even in threshold self-affine regimes, the optimal path does not fully stick on the minimal length path. As shown at the end of the previous section, for directed paths at the directed percolation threshold in $2 \mathrm{~d}$, while $\rho$ does not make sense, it is possible to describe how the distance between optimal and minimal length paths diverges. We saw that this distance is still controlled by the usual roughness exponent $\zeta$ of the paths.

At the undirected percolation threshold the self-affine solution is replaced by a fractal one, with fractal dimension equal to $D_{\min }$, the minimal length path dimension. Our results, for both hierarchical and square lattice, suggest an $\omega$ exponent close to 1 in this case. $\omega=1$ strictly would be suggested by assuming validity of the scaling relation (2.4) in the limit of fractally rough path $(\zeta \rightarrow 1)$. On the other hand we already know that eq. (2.4) does not hold at the directed percolation threshold when directed paths are considered. At the same time, in our case, $\omega$ should be a direct manifestation of the statistical geometry of minimal length paths on the $I I C$ backbone. The length fluctuations of the shortest path within the backbone are enough to determine the observed energy fluctuations. Thus, in the fractal regime of the optimal path, there is no appreciable role played by the bond energy probability distribution in the sense of determining a non-trivial stable law for minimal energy fluctuations. Minimal length fluctuations, merely due to dilution, are the dominating source of energy variance. Geometry alone seems to fully control the optimal path scaling at the percolation threshold.

We could also verify that, like in the self-affine regimes, at $1-p=p_{c}, \omega$ also characterizes 
the subleading correction to the dominant behaviour of $\overline{E_{\min }}$ and qualifies as the only scaling dimension for energy according to eq. (2.11).

Our determination of $D_{\min }$ on square lattice seems rather consistent if compared with others in the literature [10]. New interest in this kind of percolation cluster dimension has been stimulated most recently by the realization that it controls the dynamics of directed percolation interface depinning [18].

The exponent $\omega$ we determined, besides constituting, like $\rho$, an important characterization of the fractal regime of the optimal path, reflects an interesting, and, so far, unexplored, property of the backbone geometry. It would be interesting to have determinations of it in higher dimensions. Unfortunately, calculations of $\omega$ on $3 d$ Euclidean lattice are definitely out of reach with our methods.

In order to get a preliminary indication on higher $d$ situations, we decided to try an RG calculation for a hierarchical lattice, which, after removing the diagonals, coincides with what can be taken as a surrogate of $d>2$ lattice for directed problems (See Fig 3 ) [13].

The thresholds for undirected and directed bond percolation on this lattice are $p_{c}=$ $0.2760 \ldots$ and $p_{\mathrm{cd}}=0.3893 \ldots$, respectively. By the methods of section 2 we obtained for optimal self avoiding paths on this lattice: $D=1.185 \pm 0.005$ very close to the exact $D_{\min }$, and $\omega=1.16 \pm 0.05$, definitely larger than 1 .

A direction in which it is conceivable to extend the present investigation is that of including nonzero temperature effects. As already remarked above, this can be done by our methods. E.g., in the hierarchical cases, in the iteration strategy the minimal energy distribution would be replaced by a distribution for the full partition function of self-avoiding walks, $Z=\sum_{w} \exp \left(-E_{W} / k_{b} T\right)$.

For the models described in the previous sections, we expect asymptotic distributions controlling the physics at large scales to be the same as those already experienced at $T=0$. Strong disorder, $T=0$ distributions always determine the scaling, at least in the stretched phase. On the other hand, for $d \geq 3$ on Euclidean lattices, the $T=0$ strong disorder regime 
is known to extend only up to some critical temperature, marking a transition to a high $T$ regime where disorder becomes irrelevant $[19](\omega=0, \zeta=1 / 2)$. Possible candidates to show this transition, and, at the same time, the crossover to fractal regime under dilution, are hierarchical models of the type described in Fig. 3. It would be interesting, though quite laborious, to establish the effect of directedness removal and dilution on the finite temperature transition, in particular at the fractal thresholds. 


\section{References}

[1] Huse D.A. and Henley C.L., Phys. Rev. Lett. 54, 2708 (1985).

[2] Kardar M., Parisi G. and Zhang Y.C., Phys. Rev. Lett. 56, 889 (1986).

[3] Hansen A., Hinricksen E.L. and Roux S., Phys. Rev. Lett. 66, 2476 (1991)

[4] Nelson D.R., Phys. Rev. Lett. 60, 1973 (1988).

[5] See for example: Ko D.Y.K. and Seno F., Phys. Rev. E 50, R1741 (1994); Cieplak M., Maritan A. and Banavar J.R., J. Phys A27, L765 (1994).

[6] Balents L. and Kardar M. , J. Stat. Phys. 67, 1 (1992).

[7] Roux S. and Francais D, Scripta Metallurgica 25, 1087 (1991).

[8] Cao Q. and Wong P., Phys. Rev. Lett. 56, 77 (1991).

[9] K.D. Keefer and D.W. Schaefer, Phys. Rev. Lett. 56, 2376 (1986).

[10] Kinzel W. in Percolation Structures and Processes ( Annal of the Israel Physical Society 5), G. Deutscher, R. Zallen and J. Adler eds (Bristol: Adam Hilger), 425 (1983).

[11] Kardar M. and Zhang Y.C., Phys. Rev. Lett. 58, 2087 (1987).

[12] Uzelac K., Hasmy A. and Jullien R., Phys. Rev. Lett. 74, 422 (1995).

[13] Derrida B. and Griffiths R.B., Europhys. Lett. 8, 111 (1989).

[14] As an exception most recently the DDHL has been used for clarifying some properties of interfaces, in 2d random Potts ferromagnets: Kardar M., Stella A.L., Sartoni G. and Derrida B., preprint (1994).

[15] Redner S., Phys. Rev. B25, 5646 (1982) 
[16] Le Doussal P. and Machta J., J. Stat. Phys. 64, 541 (1991)

[17] Meir Y. and Harris A.B., Phys. Rev. Lett 63, 5345 (1989).

[18] Amaral L.A.N., Barabasi A.L., Buldyrev S.V., Harrington S.T., Havlin S., Sadr-Lahijany R. and Stanley H.E., SISSA database preprint: cond-mat 9412047 (1994).

[19] Derrida B. and Golinelli O. Phys. Rev A41, 4160 (1990).

[20] Cook J. and Derrida B., J. Stat. Phys. 57, 89 (1989).

[21] Derrida B., J. Phys. A14, L5 (1981).

[22] Kardar M., Phys. Rev. Lett. 55, 2235 (1985).

[23] Vanderzande C. and Komoda A., Phys.Rev. A45,R5335 (1992). 


\section{Figure Captions}

Fig 1. Iterative construction of the four hierarchical lattices: 1a) The DDHL lattice (the bonds are numbered and the paths to be considered in the iterative RG calculation are: 1,$3 ; 1,5,4 ; 2,4 ; 2,5,3$.$) ; 1b) The DHL lattice; 1c) A hierarchical lattice with p_{c}=$ $0.70830 \ldots$ and $p_{c d}=0.72578 \ldots$; 1d) A hierarchical lattice with $p_{c}=0.54984 \ldots$ and $p_{c d}=$ $0.55194 \ldots$

Fig. 2. Result of transfer matrix calculation for $\overline{\Delta E(t)}$ as a function of $\mathrm{t}$ for $L=$ $9,1-p=q=1 / 2$.

Fig. 3. Iterative construction (first step) of a "higher dimensional" hierarchical lattice. 


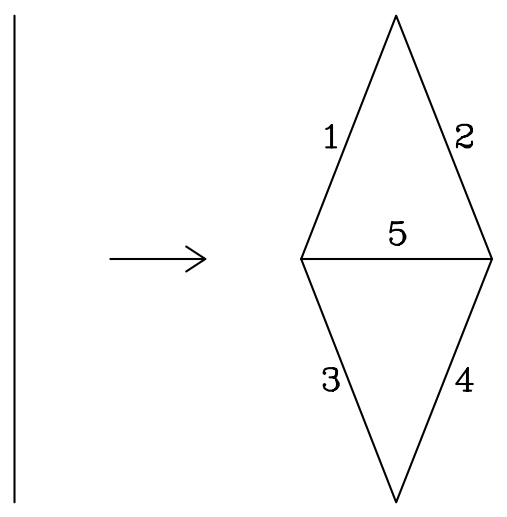

Fig. 1a

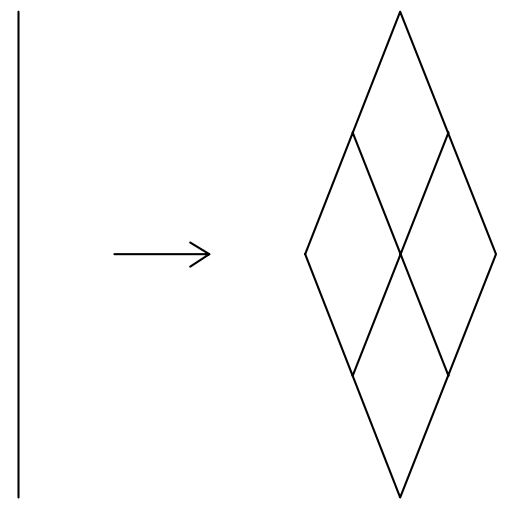

Fig. 1C

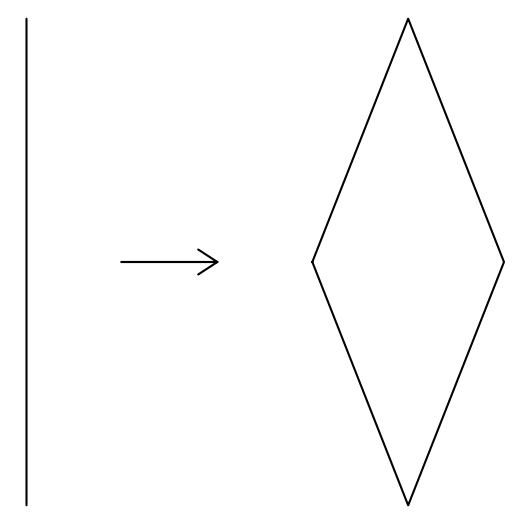

Fig. 1b

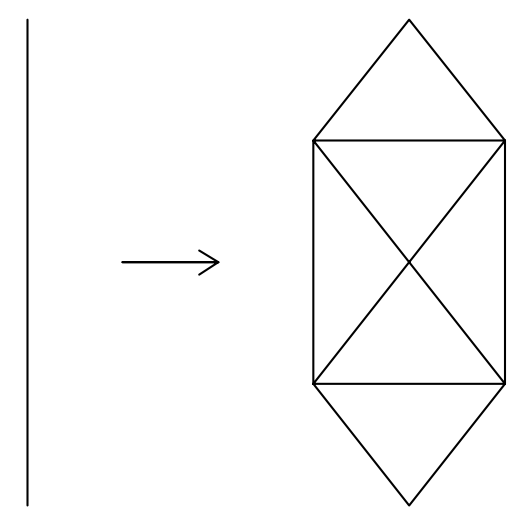

Fig. 1d 